\title{
Measurement of inclusive $\mathrm{D}^{* \pm}$ production in two-photon collisions at LEP
}

\section{L3 Collaboration}

M. Acciarri ${ }^{\mathrm{z}}$, P. Achard ${ }^{\mathrm{s}}$, O. Adriani ${ }^{\mathrm{p}}$, M. Aguilar-Benitez ${ }^{\mathrm{y}}$, J. Alcaraz ${ }^{\mathrm{y}}$, G. Alemanni " , J. Allaby ${ }^{\mathrm{q}}$, A. Aloisio ${ }^{\mathrm{ab}}$, M.G. Alviggi ${ }^{\mathrm{ab}}$, G. Ambrosi ${ }^{\mathrm{s}}$, H. Anderhub au, V.P. Andreev f,aj, T. Angelescu ', F. Anselmo i, A. Arefiev aa, T. Azemoon ${ }^{\text {c }}$, T. Aziz ${ }^{j}$, P. Bagnaia ai, L. Baksay ap, A. Balandras ${ }^{\text {d }}$, R.C. Ball ${ }^{\text {c }}$, S. Banerjee ${ }^{j}$, Sw. Banerjee ${ }^{j}$, A. Barczyk ${ }^{\text {au,as }}$, R. Barillère ${ }^{q}$, L. Barone ai P. Bartalini ${ }^{\text {v }}$, M. Basile ${ }^{\text {i }}$, R. Battiston ${ }^{\text {af }}$, A. Bay ${ }^{\text {v }}$, F. Becattini ${ }^{\mathrm{p}}$, U. Becker ${ }^{\mathrm{n}}$, F. Behner ${ }^{\text {au }}$, L. Bellucci ${ }^{\text {p }}$, J. Berdugo ${ }^{y}$, P. Berges " , B. Bertucci af, B.L. Betev ${ }^{\text {au }}$, S. Bhattacharya ${ }^{\mathrm{j}}$, M. Biasini af ${ }^{\text {, A. Biland }}{ }^{\text {au }}$, J.J. Blaising ${ }^{\mathrm{d}}$, S.C. Blyth ${ }^{\text {ag }}$, G.J. Bobbink ${ }^{\text {b }}$, A. Böhm a , L. Boldizsar ${ }^{\text {m }}$, B. Borgia ${ }^{\text {ai }}$, D. Bourilkov au,

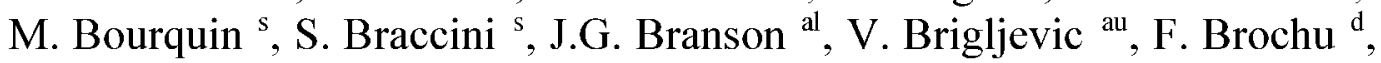
A. Buffini ${ }^{\text {p }}$, A. Buijs ${ }^{\text {aq }}$, J.D. Burger ${ }^{n}$, W.J. Burger ${ }^{\text {af }}$, J. Busenitz ${ }^{\text {ap }}$, A. Button ', X.D. Cai ", M. Campanelli ${ }^{\text {au }}$, M. Capell ", G. Cara Romeo ', G. Carlino $^{\text {ab }}$, A.M. Cartacci ${ }^{\mathrm{p}}$, J. Casaus ${ }^{\mathrm{y}}$, G. Castellini ${ }^{\mathrm{p}}$, F. Cavallari ${ }^{\text {ai }}$, N. Cavallo ${ }^{\text {ab }}$, C. Cecchi ${ }^{\text {s, }}$ M. Cerrada ${ }^{\mathrm{y}}$, F. Cesaroni ${ }^{\text {w }}$, M. Chamizo ${ }^{\mathrm{s}}$, Y.H. Chang aw , U.K. Chaturvedi ${ }^{r}$, M. Chemarin ${ }^{x}$, A. Chen ${ }^{\text {aw }}$, G. Chen ${ }^{g}$, G.M. Chen ${ }^{g}$, H.F. Chen ', H.S. Chen ${ }^{\mathrm{g}}$, X. Chereau ${ }^{\mathrm{d}}$, G. Chiefari ${ }^{\text {ab }}$, L. Cifarelli ${ }^{\text {ak }}$, F. Cindolo ${ }^{i}$, C. Civinini ${ }^{\text {}}$, I. Clare ${ }^{n}$, R. Clare ${ }^{n}$, G. Coignet ${ }^{\text {d }}$, A.P. Colijn ${ }^{b}$, N. Colino ${ }^{\mathrm{y}}, \mathrm{S}$. Costantini ${ }^{\mathrm{h}}$, F. Cotorobai ${ }^{1}$, B. Cozzoni ${ }^{\mathrm{i}}, \mathrm{B}$. de la Cruz ${ }^{\mathrm{y}}$, A. Csilling ${ }^{\mathrm{m}}$, S. Cucciarelli af , T.S. Dai ${ }^{n}$, J.A. van Dalen ${ }^{\text {ad }}$, R. D’Alessandro ${ }^{p}$, R. de Asmundis ${ }^{a b}$, P. Déglon ${ }^{\text {s }}$, A. Degré ${ }^{d}, K$. Deiters ${ }^{\text {as }}$, D. della Volpe ${ }^{a b}$, P. Denes ${ }^{\text {ah }}$, F. DeNotaristefani ${ }^{\text {ai }}$, A. De Salvo au, M. Diemoz ${ }^{\text {ai }}$, D. van Dierendonck ${ }^{\mathrm{b}}$, F. Di Lodovico ${ }^{\text {au }}$, C. Dionisi ${ }^{\text {ai }}$, M. Dittmar ${ }^{\text {au}}$, A. Dominguez ${ }^{\text {al }}$, A. Doria ${ }^{\text {ab }}$, M.T. Dova ${ }^{r, 1}$, D. Duchesneau ${ }^{d}$, D. Dufournand ${ }^{d}$, P. Duinker ${ }^{\text {b }}$, I. Duran ${ }^{\text {am }}$, H. El Mamouni ${ }^{x}$, A. Engler ${ }^{\text {ag }}$, F.J. Eppling ", F.C. Erné ${ }^{b}$, P. Extermann ${ }^{\text {s }}$, M. Fabre ${ }^{\text {as }}$, R. Faccini ai, M.A. Falagan ${ }^{y}$, S. Falciano ${ }^{\text {ai, }}$, A. Favara ${ }^{q}$, J. Fay ${ }^{x}$, O. Fedin ${ }^{\text {aj }}$, M. Felcini au, T. Ferguson ${ }^{\text {ag }}$, F. Ferroni ${ }^{\text {ai }}$, H. Fesefeldt a , E. Fiandrini af , J.H. Field ${ }^{\text {s }, ~ F . ~ F i l t h a u t ~}{ }^{\mathrm{q}}$, P.H. Fisher ${ }^{\text {n, }}$, 
I. Fisk $^{\text {al }}$, G. Forconi ${ }^{n}$, L. Fredj ${ }^{\text {s }}$, K. Freudenreich ${ }^{\text {au }}$, C. Furetta ${ }^{z}$, Yu. Galaktionov ${ }^{\text {aa,n }}$, S.N. Ganguli ${ }^{\text {i, }, ~ P . ~ G a r c i a-A b i a ~}{ }^{\text {e }}$, M. Gataullin ${ }^{\text {ae }}$, S.S. Gau ${ }^{k}$, S. Gentile ${ }^{\text {ai, }, ~}$, N. Gheordanescu ${ }^{\text {, S. Siagu }}{ }^{\text {ai }}$, Z.F. Gong ${ }^{\text {t }}$, G. Grenier ${ }^{x}$, O. Grimm ${ }^{\text {au }}$, M.W. Gruenewald ${ }^{\text {h }}$, M. Guida ${ }^{\text {ak }}$, R. van Gulik ${ }^{\text {b }, ~ V . K . ~ G u p t a ~}{ }^{\text {ah }}$, A. Gurtu ${ }^{\text {j}}$, L.J. Gutay ${ }^{a r}$, D. Haas ${ }^{\text {e }}$, A. Hasan ${ }^{\text {ac }}$, D. Hatzifotiadou ${ }^{\text {i }}$, T. Hebbeker ${ }^{\text {h }}$, A. Hervé ${ }^{\mathrm{q}}$, P. Hidas ${ }^{\mathrm{m}}$, J. Hirschfelder ${ }^{\mathrm{ag}}$, H. Hofer ${ }^{\text {au }}$, G. Holzner ${ }^{\text {au }}$, H. Hoorani ${ }^{\text {ag }}$, S.R. Hou ${ }^{\text {aw }}$, I. Iashvili at, B.N. Jin ${ }^{g}$, L.W. Jones ${ }^{c}$, P. de Jong ${ }^{b}$, I. Josa-Mutuberría ${ }^{\mathrm{y}}$, R.A. Khan ${ }^{\text {r }}$, D. Kamrad ${ }^{\text {at }}$, M. Kaur ${ }^{\text {r,2 }}$, M.N. Kienzle-Focacci ${ }^{\text {s }}$, D. Kim ${ }^{\text {ai }}$, D.H. Kim ${ }^{\text {ao }}$, J.K. $\operatorname{Kim}^{\text {ao }}$, S.C. Kim ${ }^{\text {ao }}$, J. Kirkby ${ }^{\text {q }}$, D. Kiss ${ }^{\text {m }}$, W. Kittel ${ }^{\text {ad }}$, A. Klimentov n,aa , A.C. König ad, A. Kopp ${ }^{\text {at }}$, I. Korolko ${ }^{\text {aa }}$, V. Koutsenko n,aa, M. Kräber ${ }^{\text {au }}$, R.W. Kraemer ${ }^{\text {ag }}$, W. Krenz ${ }^{\text {a }}$, A. Kunin ${ }^{\text {n,aa }}$, P. Ladron de Guevara ${ }^{\text {, }}$, I. Laktineh ${ }^{\mathrm{x}}$, G. Landi ${ }^{\mathrm{p}}$, K. Lassila-Perini ${ }^{\text {au }}$, P. Laurikainen ", A. Lavorato ${ }^{\mathrm{ak}}$, M. Lebeau ${ }^{\mathrm{q}}$, A. Lebedev ${ }^{\mathrm{n}}$, P. Lebrun ${ }^{\mathrm{x}}$, P. Lecomte ${ }^{\mathrm{au}}$, P. Lecoq ${ }^{\mathrm{q}}$, P. Le Coultre ${ }^{\mathrm{au}}$, H.J. Lee ${ }^{\mathrm{h}}$, J.M. Le Goff ${ }^{\mathrm{q}}$, R. Leiste ${ }^{\text {at }}$, E. Leonardi ${ }^{\text {ai }}$, P. Levtchenko ${ }^{\text {aj }}, \mathrm{C}$ Li $^{\mathrm{t}}$, C.H. Lin ${ }^{\text {aw }}$, W.T. Lin ${ }^{\text {aw }}$, F.L. Linde ${ }^{\text {b }}$, L. Lista ${ }^{\text {ab }}$, Z.A. Liu ${ }^{\mathrm{g}}$, W. Lohmann ${ }^{\text {at }}$, E. Longo ai, Y.S. Lu ${ }^{g}$, K. Lübelsmeyer a , C. Luci ${ }^{\text {q,ai }}$, D. Luckey ${ }^{n}$, L. Lugnier ${ }^{x}$, L. Luminari ai, W. Lustermann ${ }^{\text {au }}$, W.G. Ma ${ }^{\mathrm{t}}$, M. Maity ${ }^{\mathrm{j}}$, L. Malgeri ${ }^{\mathrm{q}}$, A. Malinin ${ }^{\text {aa, }}$, C. Maña ${ }^{y}$, D. Mangeol ${ }^{\text {ad }}$, P. Marchesini ${ }^{\text {au }}$, G. Marian ${ }^{o}$, J.P. Martin x , F. Marzano ai, G.G.G. Massaro ${ }^{\text {b }}$, K. Mazumdar ${ }^{\mathrm{j}}$, R.R. McNeil ${ }^{\mathrm{f}}$, S. Mele ${ }^{q}$, L. Merola ${ }^{\text {ab }}$, M. Meschini ${ }^{p}$, W.J. Metzger ad ${ }^{\text {ad }}$ M. von der Mey ${ }^{a}$, A. Mihul ${ }^{1}$, H. Milcent ${ }^{\mathrm{q}}$, G. Mirabelli ai, J. Mnich ${ }^{\mathrm{q}}$, G.B. Mohanty ${ }^{\mathrm{j}}$, P. Molnar ${ }^{\mathrm{h}}$, B. Monteleoni ${ }^{\mathrm{p}, 3}$, T. Moulik j, G.S. Muanza ${ }^{\mathrm{x}}$, F. Muheim ${ }^{\mathrm{s}}$, A.J.M. Muijs ${ }^{\mathrm{b}}$, M. Musy ${ }^{\text {ai }}$, M. Napolitano ${ }^{\text {ab }}$, F. Nessi-Tedaldi au, H. Newman ${ }^{\text {ae }}$, T. Niessen ${ }^{\text {a }}$, A. Nisati ai, H. Nowak at, Y.D. Oh ${ }^{\text {ao }}$, G. Organtini ai, R. Ostonen ", C. Palomares ${ }^{y}$, D. Pandoulas ${ }^{\text {a }}$, S. Paoletti ${ }^{\text {ai, }}$, P. Paolucci ${ }^{\text {ab }}$, R. Paramatti ${ }^{\text {ai }}$, H.K. Park ${ }^{\text {ag }}$, I.H. Park ${ }^{\text {ao }}$, G. Pascale ${ }^{\text {ai }}$, G. Passaleva ${ }^{\mathrm{q}}$, S. Patricelli ${ }^{\mathrm{ab}}$, T. Paul ${ }^{\mathrm{k}}$, M. Pauluzzi af C. Paus ${ }^{q}$, F. Pauss ${ }^{\text {au }}$, D. Peach ${ }^{\mathrm{q}}$, M. Pedace ${ }^{\text {ai }}$, S. Pensotti $^{z}$, D. Perret-Gallix ${ }^{d}$, B. Petersen ${ }^{\text {ad }}$, D. Piccolo ${ }^{\text {ab }}$, F. Pierella ${ }^{i}$, M. Pieri ${ }^{\text {p }}$, P.A. Piroué ${ }^{\text {ah }}$, E. Pistolesi ${ }^{z}$, V. Plyaskin aa, M. Pohl au, V. Pojidaev aa, , H. Postema ${ }^{\text {n }}$, J. Pothier ${ }^{\mathrm{q}}$, N. Produit ${ }^{\mathrm{s}}$,

D.O. Prokofiev ar , D. Prokofiev aj, J. Quartieri ak, G. Rahal-Callot ${ }^{\mathrm{au}, \mathrm{q}}$, M.A. Rahaman ${ }^{j}$, P. Raics ${ }^{\circ}$, N. Raja ${ }^{j}$, R. Ramelli au, P.G. Rancoita ${ }^{z}$, G. Raven ${ }^{\text {al }}$, P. Razis ${ }^{\text {ac }}$, D. Ren ${ }^{\text {au }}$, M. Rescigno ${ }^{\text {ai }}$, S. Reucroft ${ }^{\text {k, }}$ T. van Rhee ${ }^{\text {aq }}$, S. Riemann ${ }^{\text {at }}$, K. Riles ${ }^{c}$, A. Robohm ${ }^{\text {au }}$, J. Rodin ${ }^{\text {ap }}$, B.P. Roe ${ }^{c}$, L. Romero ${ }^{y}$, A. Rosca ${ }^{\text {h, }}$, S. Rosier-Lees ${ }^{\text {d, J.A. Rubio }}{ }^{\text {}}$, D. Ruschmeier ${ }^{\text {h }}$, H. Rykaczewski ${ }^{\text {au }}$, S. Sarkar ai, J. Salicio $^{\text {q }, ~ E . ~ S a n c h e z ~}{ }^{\text {q }}$, M.P. Sanders ${ }^{\text {ad }}$, M.E. Sarakinos ", C. Schäfer ${ }^{\text {a }}$, V. Schegelsky aj, S. Schmidt-Kaerst ${ }^{\text {a }}$, D. Schmitz ${ }^{\text {a }}$, H. Schopper ${ }^{\text {av }}$, D.J. Schotanus ${ }^{\text {ad }}$, G. Schwering ${ }^{\text {a }}$, C. Sciacca ${ }^{\text {ab }}$, D. Sciarrino ${ }^{\text {s }}$, A. Seganti ${ }^{\mathrm{i}}$, L. Servoli af , S. Shevchenko ${ }^{\text {ae }}$, N. Shivarov ${ }^{\text {an }}$, V. Shoutko ${ }^{\text {aa }}$, E. Shumilov aa, 
A. Shvorob ${ }^{\text {ae }}$, T. Siedenburg ${ }^{\text {a }}$, D. Son ${ }^{\text {ao }}$, B. Smith ${ }^{\text {ag }}$, P. Spillantini ${ }^{\mathrm{p}}$, M. Steuer ${ }^{\mathrm{n}}$, D.P. Stickland ${ }^{\text {ah }}$, A. Stone ${ }^{\mathrm{f}}$, H. Stone ${ }^{\text {ah,3 }}$, B. Stoyanov ${ }^{\text {an }}$, A. Straessner ${ }^{\text {a }}$, K. Sudhakar ${ }^{j}$, G. Sultanov ${ }^{\text {r }}$, L.Z. Sun ${ }^{\text {t }}$, H. Suter ${ }^{\text {au }}$, J.D. Swain ${ }^{\mathrm{r}}$, Z. Szillasi ${ }^{\text {ap,4 }}$, T. Sztaricshai ap,4 X.W. Tang ${ }^{\mathrm{g}}$, L. Tauscher ${ }^{\mathrm{e}}$, L. Taylor ${ }^{\mathrm{k}}, \mathrm{C}$. Timmermans ${ }^{\text {ad }}$, Samuel C.C. Ting ", S.M. Ting ${ }^{n}$, S.C. Tonwar ${ }^{\mathrm{j}}$, J. Tóth ${ }^{\mathrm{m}}$, C. Tully ${ }^{\text {ah }}$, K.L. Tung ${ }^{\mathrm{g}}$, Y. Uchida ${ }^{\mathrm{n}}$, J. Ulbricht ${ }^{\mathrm{au}}$, E. Valente ${ }^{\text {ai }}$, G. Vesztergombi ${ }^{\mathrm{m}}$, I. Vetlitsky ${ }^{a}$, D. Vicinanza ${ }^{\mathrm{ak}}$, G. Viertel ${ }^{\text {au }}, \mathrm{S}$. Villa ${ }^{\mathrm{k}}$, M. Vivargent ${ }^{\mathrm{d}}$, S. Vlachos ${ }^{\mathrm{e}}$, I. Vodopianov aj, H. Vogel ${ }^{\text {ag }}, \mathrm{H}$. Vogt ${ }^{\text {at }}$, I. Vorobiev ${ }^{\text {aa }}$, A.A. Vorobyov ${ }^{\text {aj, }}$, A. Vorvolakos ${ }^{\text {ac }}$, M. Wadhwa ${ }^{e}$, W. Wallraff ${ }^{a}$, M. Wang ${ }^{n}$, X.L. Wang ${ }^{t}$, Z.M. Wang ${ }^{\mathrm{t}}$, A. Weber ${ }^{\mathrm{a}}$, M. Weber ${ }^{\mathrm{a}}$, P. Wienemann ${ }^{\mathrm{a}}$, H. Wilkens ${ }^{\text {ad }}$, S.X. Wu ${ }^{\mathrm{n}}$, S. Wynhoff ${ }^{a}$, L. Xia ${ }^{\text {ae }}$, Z.Z. Xu ${ }^{\text {}}$, B.Z. Yang ${ }^{\text {t }}$, C.G. Yang ${ }^{\mathrm{g}}$, H.J. Yang ${ }^{\mathrm{g}}$, M. Yang ${ }^{\mathrm{g}}$, J.B. Ye ${ }^{\mathrm{t}}$, S.C. Yeh ${ }^{\mathrm{ax}}$, An. Zalite ${ }^{\text {aj }}$, Yu. Zalite ${ }^{\text {aj }}$, Z.P. Zhang ${ }^{\mathrm{t}}$, G.Y. Zhu ${ }^{\mathrm{g}}$, R.Y. Zhu ${ }^{\text {ae }}$, A. Zichichi ${ }^{\mathrm{i}, \mathrm{q}, \mathrm{r}}$, F. Ziegler ${ }^{\text {at }}$, G. Zilizi ${ }^{\text {ap,4 }}$, M. Zöller ${ }^{\text {a }}$

${ }^{a}$ I. Physikalisches Institut, RWTH, D-52056 Aachen, Germany, and III. Physikalisches Institut, RWTH, D-52056 Aachen, Germany ${ }^{5}$

${ }^{\mathrm{b}}$ National Institute for High Energy Physics, NIKHEF, and University of Amsterdam, NL-1009 DB Amsterdam, The Netherlands ${ }^{c}$ University of Michigan, Ann Arbor, $M I$ 48109, USA

${ }^{\mathrm{d}}$ Laboratoire d'Annecy-le-Vieux de Physique des Particules, LAPP, IN2P3-CNRS, BP 110, F-74941 Annecy-le-Vieux CEDEX, France

${ }^{\mathrm{e}}$ Institute of Physics, University of Basel, CH-4056 Basel, Switzerland

${ }^{\mathrm{f}}$ Louisiana State University, Baton Rouge, LA 70803, USA

Institute of High Energy Physics, IHEP, 100039 Beijing, China ${ }^{6}$

${ }^{\mathrm{h}}$ Humboldt University, D-10099 Berlin, Germany ${ }^{5}$

${ }^{\mathrm{i}}$ University of Bologna and INFN-Sezione di Bologna, I-40126 Bologna, Italy

${ }^{\mathrm{j}}$ Tata Institute of Fundamental Research, Bombay 400005 , India

${ }^{\mathrm{k}}$ Northeastern University, Boston, MA 02115, USA

${ }^{1}$ Institute of Atomic Physics and University of Bucharest, $R-76900$ Bucharest, Romania

${ }^{\mathrm{m}}$ Central Research Institute for Physics of the Hungarian Academy of Sciences, H-1525 Budapest 114, Hungary ${ }^{7}$

${ }^{\mathrm{n}}$ Massachusetts Institute of Technology, Cambridge, MA 02139, USA

${ }^{\circ}$ Lajos Kossuth University-ATOMKI, H-4010 Debrecen, Hungary ${ }^{4}$

${ }^{\mathrm{p}}$ INFN Sezione di Firenze and University of Florence, I-50125 Florence, Italy

${ }^{q}$ European Laboratory for Particle Physics, CERN, CH-1211 Geneva 23, Switzerland

${ }^{\mathrm{r}}$ World Laboratory, FBLJA Project, CH-1211 Geneva 23, Switzerland

s University of Geneva, $\mathrm{CH}-1211$ Geneva 4, Switzerland

${ }^{\mathrm{t}}$ Chinese University of Science and Technology, USTC, Hefei, Anhui 230 029, China ${ }^{6}$

" SEFT, Research Institute for High Energy Physics, P.O. Box 9, SF-00014 Helsinki, Finland

${ }^{\mathrm{V}}$ University of Lausanne, CH-1015 Lausanne, Switzerland

${ }^{\mathrm{w}}$ INFN-Sezione di Lecce and Universitá Degli Studi di Lecce, I-73100 Lecce, Italy

${ }^{x}$ Institut de Physique Nucléaire de Lyon, IN2P3-CNRS, Université Claude Bernard, F-69622 Villeurbanne, France

${ }^{y}$ Centro de Investigaciones Energéticas, Medioambientales y Tecnologicas, CIEMAT, E-28040 Madrid, Spain ${ }^{8}$

${ }^{\mathrm{I}}$ INFN-Sezione di Milano, I-20133 Milan, Italy

anstitute of Theoretical and Experimental Physics, ITEP, Moscow, Russia

${ }^{\mathrm{ab}}$ INFN-Sezione di Napoli and University of Naples, I-80125 Naples, Italy

ac Department of Natural Sciences, University of Cyprus, Nicosia, Cyprus

${ }^{\text {ad }}$ University of Nijmegen and NIKHEF, NL-6525 ED Nijmegen, The Netherlands

${ }^{\text {ae }}$ California Institute of Technology, Pasadena, CA 91125, USA

af INFN-Sezione di Perugia and Universitá Degli Studi di Perugia, I-06100 Perugia, Italy

ag Carnegie Mellon University, Pittsburgh, PA 15213, USA

ah Princeton University, Princeton, NJ 08544, USA

ai INFN-Sezione di Roma and University of Rome, "La Sapienza", I-00185 Rome, Italy

${ }^{\text {aj }}$ Nuclear Physics Institute, St. Petersburg, Russia 
ak University and INFN, Salerno, I-84100 Salerno, Italy

al University of California, San Diego, CA 92093, USA

am Dept. de Fisica de Particulas Elementales, Univ. de Santiago, E-15706 Santiago de Compostela, Spain

an Bulgarian Academy of Sciences, Central Lab. of Mechatronics and Instrumentation, BU-1113 Sofia, Bulgaria

ao Center for High Energy Physics, Adv. Inst. of Sciences and Technology, 305-701 Taejon, South Korea

ap University of Alabama, Tuscaloosa, AL 35486, USA

${ }^{a q}$ Utrecht University and NIKHEF, NL-3584 CB Utrecht, The Netherlands

${ }^{\text {ar }}$ Purdue University, West Lafayette, IN 47907, USA

as Paul Scherrer Institut, PSI, CH-5232 Villigen, Switzerland

${ }^{a t}$ DESY, D-15738 Zeuthen, Germany

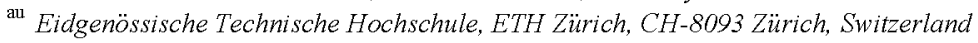

av University of Hamburg, D-22761 Hamburg, Germany

${ }^{\text {aw }}$ National Central University, Chung-Li, Taiwan, ROC

${ }^{a x}$ Department of Physics, National Tsing Hua University, Taiwan, ROC

Received 26 July 1999; accepted 24 September 1999

Editor: K. Winter

\begin{abstract}
Inclusive production of $\mathrm{D}^{* \pm}$ mesons in two-photon collisions was measured by the L3 experiment at LEP. The data were collected at a centre-of-mass energy $\sqrt{s}=189 \mathrm{GeV}$ with an integrated luminosity of $176.4 \mathrm{pb}^{-1}$. Differential cross sections of the process $\mathrm{e}^{+} \mathrm{e}^{-} \rightarrow \mathrm{e}^{+} \mathrm{e}^{-} \mathrm{D}^{* \pm} \mathrm{X}$ are determined as functions of the transverse momentum and pseudorapidity of the $\mathrm{D}^{* \pm}$ mesons in the kinematic region $1 \mathrm{GeV}<p_{T}^{\mathrm{D}^{*}}<5 \mathrm{GeV}$ and $\left|\eta^{\mathrm{D}^{*}}\right|<1.4$. The cross section integrated over this phase space domain is measured to be $132 \pm 22$ (stat.) \pm 26 (syst.) pb. The differential cross sections are compared with next-to-leading order perturbative QCD calculations. (c) 1999 Published by Elsevier Science B.V. All rights reserved.
\end{abstract}

\section{Introduction}

The study of charm production in two-photon collisions provides a means for testing perturbative QCD and for probing the gluon content of the photon [1]. Charmed quarks can be produced in "directphoton" processes, in which the interacting photons

\footnotetext{
${ }^{1}$ Also supported by CONICET and Universidad Nacional de La Plata, CC 67, 1900 La Plata, Argentina.

${ }^{2}$ Also supported by Panjab University, Chandigarh-160014, India.

${ }^{3}$ Deceased.

${ }^{4}$ Also supported by the Hungarian OTKA fund under contract numbers T22238 and T026178.

${ }^{5}$ Supported by the German Bundesministerium für Bildung, Wissenschaft, Forschung und Technologie.

${ }^{6}$ Supported by the National Natural Science Foundation of China.

${ }^{7}$ Supported by the Hungarian OTKA fund under contract numbers T019181, F023259 and T024011.

${ }^{8}$ Supported also by the Comisión Interministerial de Ciencia y Tecnología.
}

behave as point-like particles and couple directly to a charmed quark pair. Another class of processes contributing to the charm production are the "resolvedphoton" processes, where one or both interacting photons fluctuate into a flux of partons. In the "single resolved-photon" processes the unresolved photon interacts with a constituent parton from the resolved photon, whereas in the "double resolved-photon", processes a hard scattering between the constituent partons of the two resolved photons takes place. In the next-to-leading order QCD only the sum of direct and resolved-photon processes is unambiguously defined. The experimental measurement of differential cross sections for production of open charmed particles allows a detailed investigation of the charm production mechanism.

Charm production in two-photon collisions has been measured at lower centre-of-mass energies at PEP, PETRA, TRISTAN and LEP [2-8], identifying charmed quarks by detecting $\mathrm{D}^{* \pm}$ mesons, soft pions, inclusive leptons and $\mathrm{K}_{\mathrm{S}}^{0}$ mesons. In a previous measurement by the L3 experiment [9], events 
containing charmed quarks were tagged by detecting electrons and muons from semileptonic decays of charmed hadrons. In the present study charmed vector mesons $D^{*}(2010)^{ \pm}$are identified by the small energy released in $\mathrm{D}^{*}$ decay, applying the mass difference technique [10] to the decay chains ${ }^{9}$

$$
\begin{aligned}
\mathrm{D}^{*+} \rightarrow & \mathrm{D}^{0} \pi_{\mathrm{S}}^{+} \\
& \hookrightarrow \mathrm{K}^{-} \pi^{+} \\
& \hookrightarrow \mathrm{K}^{-} \pi^{+} \pi^{0}
\end{aligned}
$$

The presence of a low-momentum, "soft" pion, $\pi_{\mathrm{S}}^{+}$, ensures that the resolution of the mass difference $M\left(\mathrm{D}^{0} \pi_{\mathrm{S}}^{+}\right)-M\left(\mathrm{D}^{0}\right)$ is superior to the resolution of the reconstructed $\mathrm{D}^{0}$ and $\mathrm{D}^{*+}$ masses themselves. The $\mathrm{D}^{*+}$ signal appears as a narrow peak close to the kinematic threshold in the mass difference distributions $M\left(\mathrm{~K}^{-} \pi^{+} \pi_{\mathrm{S}}^{+}\right)-M\left(\mathrm{~K}^{-} \pi^{+}\right)$and $M\left(\mathrm{~K}^{-} \pi^{+} \pi^{0} \pi_{\mathrm{S}}^{+}\right)-M\left(\mathrm{~K}^{-} \pi^{+} \pi^{0}\right)$. The combined branching fractions are $\mathrm{BR}\left(\mathrm{D}^{*+} \rightarrow \mathrm{D}^{0} \pi_{\mathrm{S}}^{+}\right) \cdot \mathrm{BR}\left(\mathrm{D}^{0}\right.$ $\left.\rightarrow \mathrm{K}^{-} \pi^{+}\right)=0.0263 \pm 0.0008$ and $\mathrm{BR}\left(\mathrm{D}^{*+} \rightarrow\right.$ $\left.\mathrm{D}^{0} \pi_{\mathrm{S}}^{+}\right) \cdot \mathrm{BR}\left(\mathrm{D}^{0} \rightarrow \mathrm{K}^{-} \pi^{+} \pi^{0}\right)=0.0949 \pm 0.0064$, as given in Ref. [11].

\section{Selection of hadronic two-photon events}

The data were collected by the L3 detector [12] at LEP in 1998 at a centre-of-mass energy $\sqrt{\mathrm{s}}=$ $189 \mathrm{GeV}$. The integrated luminosity is $176.4 \mathrm{pb}^{-1}$.

For efficiency studies, samples of $\mathrm{e}^{+} \mathrm{e}^{-} \rightarrow$ $\mathrm{e}^{+} \mathrm{e}^{-} \gamma^{*} \gamma^{*} \rightarrow \mathrm{e}^{+} \mathrm{e}^{-} \mathrm{c} \overline{\mathrm{c} X}$ events are generated using the PYTHIA [13] and the JAMVG [14] Monte Carlo generators. The background sources are simulated by JAMVG $\left(\mathrm{e}^{+} \mathrm{e}^{-} \rightarrow \mathrm{e}^{+} \mathrm{e}^{-} \tau^{+} \tau^{-}\right)$, KORALZ [15] $\left(\mathrm{e}^{+} \mathrm{e}^{-} \rightarrow \tau^{+} \tau^{-}(\gamma)\right)$, KORALW [16] $\left(\mathrm{e}^{+} \mathrm{e}^{-} \rightarrow\right.$ $\left.\mathrm{W}^{+} \mathrm{W}^{-} \rightarrow \mathbf{f} \overline{\mathrm{f}}^{\prime} \mathrm{f} \overline{\mathrm{f}}^{\prime}\right)$ and PYTHIA $\left(\mathrm{e}^{+} \mathrm{e}^{-} \rightarrow \mathrm{q} \overline{\mathrm{q}}(\gamma)\right.$, $\left.\mathrm{e}^{+} \mathrm{e}^{-} \rightarrow \mathrm{e}^{+} \mathrm{e}^{-} \mathrm{q} \overline{\mathrm{q}}\right)$. The Monte Carlo events are processed in the same way as the data.

Reconstruction of the decay chains (1) and (2) requires a sample of events containing hadronic final states. Events of the type $\mathrm{e}^{+} \mathrm{e}^{-} \rightarrow \mathrm{e}^{+} \mathrm{e}^{-} \gamma^{*} \gamma^{*} \rightarrow$ $\mathrm{e}^{+} \mathrm{e}^{-}$hadrons are selected by cuts on the energy

\footnotetext{
${ }^{9}$ The charge conjugate reactions are included throughout the paper.
}

measured in the electromagnetic and hadron calorimeters and using tracking information. To exclude annihilation events, the total visible energy must not exceed $0.4 \sqrt{s}$, the energy deposited in the electromagnetic calorimeter must be less than $30 \mathrm{GeV}$ and the energy in the hadron calorimeter less than $40 \mathrm{GeV}$. The transverse component of the missing momentum vector must be less than $10 \mathrm{GeV}$ and the value of the event thrust must be smaller than 0.95 . Events are required to have at least three charged particles reconstructed in the tracking chamber.

A total of 1253890 events pass the hadron selection cuts. The contamination from annihilation processes and two-photon production of tau pairs is less than $0.5 \%$. The subsequent reconstruction, which forms $\mathrm{D}^{*+}$ candidates from three-prong decays with invariant mass exceeding $2 \mathrm{GeV}$, suppresses these background contributions to a negligible level.

The trigger efficiency for detecting two-photon hadronic final states is $(87 \pm 3) \%$, determined from the data sample itself using a set of independent triggers.

\section{Mass reconstruction of $\mathbf{D}^{*+}$ decays}

The identification of $\mathrm{D}^{*+}$ mesons proceeds through two steps: selection of $\mathrm{D}^{0}$ candidates, which are then combined with another track to form $\mathrm{D}^{*+}$ candidates.

Tracks are used for reconstruction of $\mathrm{D}^{0}$ decays if they satisfy the following requirements:

- Transverse momentum greater than $150 \mathrm{MeV}$.

- At least 40 wire hits measured by the tracking chamber.

- Distance of closest approach to the event vertex smaller than $1 \mathrm{~mm}$ in the transverse plane

A pair of tracks of opposite charge is required to pass the following criteria in order to be considered as a $\mathrm{K}^{-} \pi^{+}$system from a $\mathrm{D}^{0}$ decay:

- The intersection point of the tracks in the transverse plane must be displaced by no more than 3 $\mathrm{mm}$ away from the event vertex.

- $P_{\mathrm{K}} \cdot P_{\pi}>2 \cdot 10^{-3}$, where $P_{\mathrm{K}}$ and $P_{\pi}$ are the probabilities, calculated from the measured energy loss $d E / d X$ of each track, for kaon and pion mass hypotheses of the corresponding tracks. 
The selection of tracks and their combinations into neutral pairs is identical for the channels (1) and (2) in order to minimize the relative systematic error between the two decay modes.

To reconstruct $\mathrm{D}^{0}$ decays in the $\mathrm{K}^{-} \pi^{+} \pi^{0}$ decay mode, a neutral pion is added to the selected $\mathrm{K}^{-} \pi^{+}$ system. Neutral pion candidates are formed by a pair of photons, identified as isolated clusters in the electromagnetic calorimeter, not matched with a charged track. Photons are accepted for $\pi^{0}$ reconstruction if they are detected in the barrel part of the electromagnetic calorimeter and their energies are greater than $100 \mathrm{MeV}$. The $\pi^{0}$ candidates must have the invariant mass of the photon pair in the mass window of $\pm 15 \mathrm{MeV}$ around the $\pi^{0}$ mass. The decay $\mathrm{D}^{0} \rightarrow \mathrm{K}^{-} \pi^{+} \pi^{0}$ proceeds dominantly through one of the quasi-two-body intermediate states $\overline{\mathrm{K}}^{* 0} \pi^{0}, \mathrm{~K}^{*-} \pi^{+}$and $\mathrm{K}^{-} \rho^{+}$[11]. We require either the invariant mass of a $\mathrm{K} \pi$ subsystem to be within $\pm 80 \mathrm{MeV}$ of the corresponding $\mathrm{K}^{*}(892)$ mass or the invariant mass of the $\pi^{+} \pi^{0}$ system to be within $\pm 150 \mathrm{MeV}$ of the $\rho^{+}$mass. If this condition is met for a given intermediate resonant state, we make use of the P-wave properties of a vector particle decay into two scalar particles and demand in addition the helicity angle $\theta^{*}$ of the corresponding decay cascade to satisfy the condition $\left|\cos \theta^{*}\right|>0.4$. The helicity angle $\theta^{*}$ is defined as the angle between the direction of a decay product of the vector resonance $\left(\overline{\mathrm{K}}^{* 0}, \mathrm{~K}^{*-}\right.$ or $\left.\rho^{+}\right)$and the direction of the pseudoscalar particle $\left(\pi^{0}, \pi^{+}\right.$or $\left.\mathrm{K}^{-}\right)$from the $\mathrm{D}^{0}$ decay, calculated in the intermediate resonance rest frame.

To reduce the combinatorial background when reconstructing $\mathrm{D}^{0}$ decays into the $\mathrm{K}^{-} \pi^{+}$final state, the opening angle of the track pair in space must be smaller than $2.5 \mathrm{rad}$. The combinatorial background for the $\mathrm{K}^{-} \boldsymbol{\pi}^{+} \boldsymbol{\pi}^{0}$ decay mode is suppressed by requiring the solid angle, defined by the directions of flight of the three decay products, to be smaller than 2 srad.

The invariant mass of the $\mathrm{K}^{-} \pi^{+}$system is calculated and if it is in the range of $\pm 100 \mathrm{MeV}$ around the mass of the $\mathrm{D}^{0}$ mesons [11], the combination is retained as a $\mathrm{D}^{0}$ candidate for the decay channel (1). The corresponding mass window for candidates in channel (2) is $\pm 50 \mathrm{MeV}$. The different mass windows reflect the corresponding $\mathrm{D}^{0}$ mass resolutions, as obtained by Monte Carlo studies. The better resolution of the $D^{0}$ reconstruction in channel (2) is due to the softer and thus better measured charged particles produced in the three body decay and to the use of a well-measured $\pi^{0}$.

Finally, the probability that a particular $\mathrm{K}^{-} \pi^{+}$ combination comes from a $\mathrm{D}^{0}$ decay in channel (1) is determined from a $1 \mathrm{C}$ kinematic fit, in which the invariant mass of the pair is constrained to the $\mathrm{D}^{0}$ mass. For the $\mathrm{K}^{-} \pi^{+} \pi^{0}$ final state we perform a $2 \mathrm{C}$ fit, constraining the mass of the whole system to the $\mathrm{D}^{0}$ mass and the two-photon mass to the $\pi^{0}$ mass. A combination is accepted as a $\mathrm{D}^{0}$ candidate if the confidence level of the fit is greater than $0.5 \%$.

In the second step of the $\mathrm{D}^{*+}$ reconstruction, we consider all combinations of a given $\mathrm{D}^{0}$ candidate with an additional track of positive charge, assumed to be the soft pion $\pi_{S}^{+}$, resulting from the $D^{*+}$ decay. A track used as a soft pion candidate must have a transverse momentum greater than $50 \mathrm{MeV}$, at least 25 wire hits measured by the tracking chamber, and a distance of closest approach to the event vertex smaller than $3 \mathrm{~mm}$ in the transverse plane.
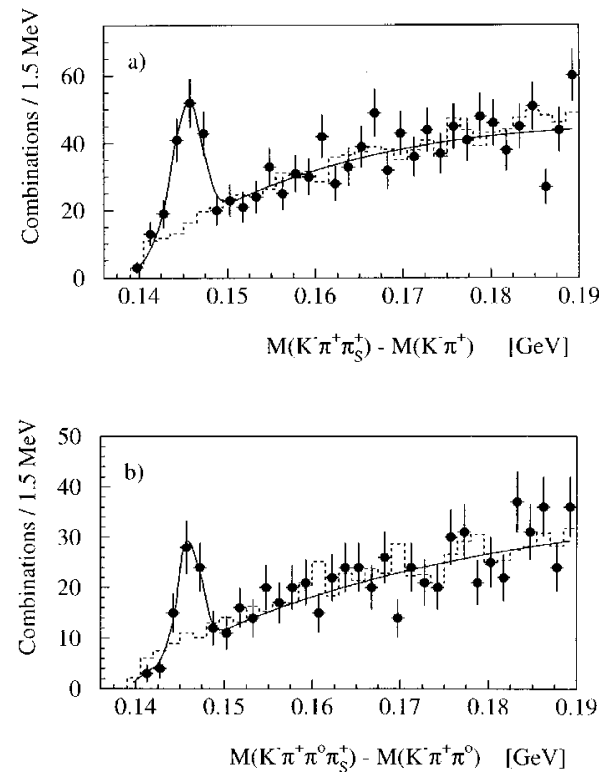

Fig. 1. Mass difference distribution for $\mathrm{D}^{0}$ decays into (a) $\mathrm{K}^{-} \pi^{+}$ and (b) $\mathrm{K}^{-} \pi^{+} \pi^{0}$. The points are data, the line is the result of the fit to the data points used to evaluate the $\mathrm{D}^{*+}$ signal and the dashed histogram represents a background check, see the text. 
A cut on the transverse momentum of the $\mathrm{D}^{0} \pi_{\mathrm{S}}^{+}$ system, $p_{T}>1 \mathrm{GeV}$, is imposed in order to exclude the region of small acceptance of $\mathrm{D}^{*+}$.

The mass difference distribution $\Delta M=$ $M\left(\mathrm{D}^{0} \pi_{\mathrm{S}}^{+}\right)-M\left(\mathrm{D}^{0}\right)$ for the selected $\mathrm{D}^{0} \pi_{\mathrm{S}}^{+}$combinations in the two channels is shown in Fig. 1. The contributions from the decay cascades (1) and (2) accumulate in narrow peaks close to the kinematic threshold. The mass difference spectrum is fitted by a sum of a Gaussian function for the signal and a term for the background of the form $\sum_{i=1}^{2} a_{i}(\Delta M-$ $\left.m_{\pi}\right)^{b_{i}}$, where $a_{i}$ and $b_{i}$ are free parameters. The peak positions, determined by the fit, are $145.5 \pm$ $0.2 \mathrm{MeV}$ for the channel (1) and $146.1 \pm 0.3 \mathrm{MeV}$ for the channel (2), and agree well with the world average value for the mass difference $m_{\mathrm{D}^{++}}-m_{\mathrm{D}^{0}}$ [11]. The good description of the background by the fit is corroborated by a background estimate obtained from the data themselves employing an event-mixing technique. For this, $\mathrm{D}^{0}$ candidates from a given event are combined with soft tracks from another event, containing $\mathrm{D}^{0}$ candidates. The resulting background distributions are normalized to the data distributions in the region $\Delta M>0.152 \mathrm{GeV}$ and shown in Fig. 1 by the dashed histograms. The number of reconstructed $\mathrm{D}^{*} \pm$ mesons is taken to be the number of observed entries in the signal region $0.141 \mathrm{GeV}<\Delta M<0.150 \mathrm{GeV}$, less the integral of the background fit component over that region. The

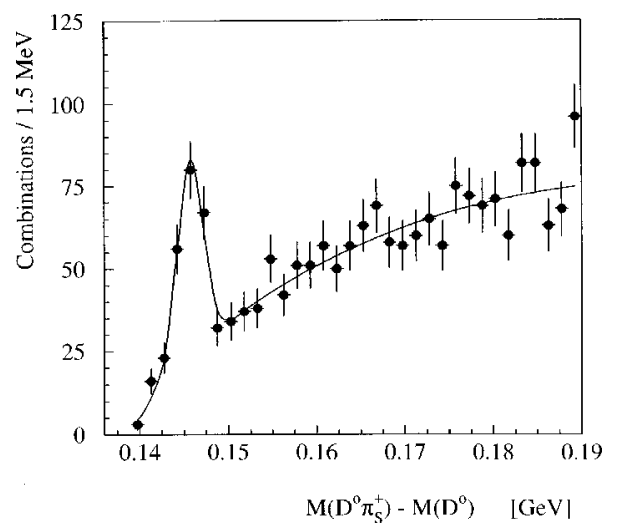

Fig. 2. Combined mass difference distribution for $\mathrm{D}^{0}$ decay channels $\mathrm{K}^{-} \boldsymbol{\pi}^{+}$and $\mathrm{K}^{-} \boldsymbol{\pi}^{+} \boldsymbol{\pi}^{0}$. The points are data and the line is the result of the fit used to evaluate the $\mathrm{D}^{*+}$ signal.
$\mathrm{D}^{*} \pm$ signal is estimated to be $102 \pm 17$ events in channel (1) and $42 \pm 11$ in channel (2).

The combinatorial multiplicity in the signal regions $\Delta M<0.150 \mathrm{GeV}$ is $1.04 \pm 0.01$ for the channel (1) and $1.05 \pm 0.02$ for the channel (2). There is no overlap of events in this region between the two channels and since the corresponding peak positions of the $D^{* \pm}$ signal agree well, we add the two distributions shown in Fig. 1 and the resulting mass difference spectrum is shown in Fig. 2. The total number of the observed $D^{*} \pm$ mesons, obtained from the fit to the combined spectrum, is $149 \pm 20$. If the combined spectrum is split into two distributions for negative and positive charmed events, the fit result is $66 \pm 14 \mathrm{D}^{*-}$ mesons and $76 \pm 15 \mathrm{D}^{*+}$ mesons.

\section{Inclusive $D^{*} \pm$ production cross section}

The cross section of inclusive $\mathrm{D}^{*} \pm$ production is determined for the whole two-photon centre of mass range, from charm threshold to the maximum accessible by the beam energy, with no cut on photon virtuality (no anti-tag condition). The cross sections, summed over $\mathrm{D}^{*-}$ and $\mathrm{D}^{*+}$, are given only in the visible kinematic region of experimental acceptance, to avoid model-dependent extrapolation uncertainties. In the present analysis, the selection cuts and the available statistics allow to cover the following phase space domain of $D^{* \pm}$ pseudorapidity $\left|\eta^{D^{*}}\right|$ and transverse momentum $p_{T}^{\mathrm{D}^{*}}$ :

$\left|\eta^{\mathrm{D}^{*}}\right|<1.4, \quad 1 \mathrm{GeV}<p_{T^{*}}^{\mathrm{D}^{*}}<5 \mathrm{GeV}$.

The differential spectra are obtained by fits to the mass difference distributions subdivided into three intervals of $p_{T}^{\mathrm{D}^{*}}$ or $\left|\eta^{\mathrm{D}^{*}}\right|$, the other variable being integrated over its kinematic region. Based on Monte Carlo studies, the resolution of the reconstructed $p_{T}^{\mathrm{D}^{*}}$ is determined to be about $30 \mathrm{MeV}$ and the resolution of $\left|\eta^{D^{*}}\right|$ about 0.008 units of pseudorapidity. Thus the smearing and the resulting event migration between adjacent bins in the spectra of the reconstructed $\mathrm{D}^{* \pm}$ mesons is negligible.

The efficiencies for the reconstruction of $D^{*} \pm$ mesons are calculated separately for direct-photon processes and for single resolved-photon processes with Monte Carlo events generated by the PYTHIA 

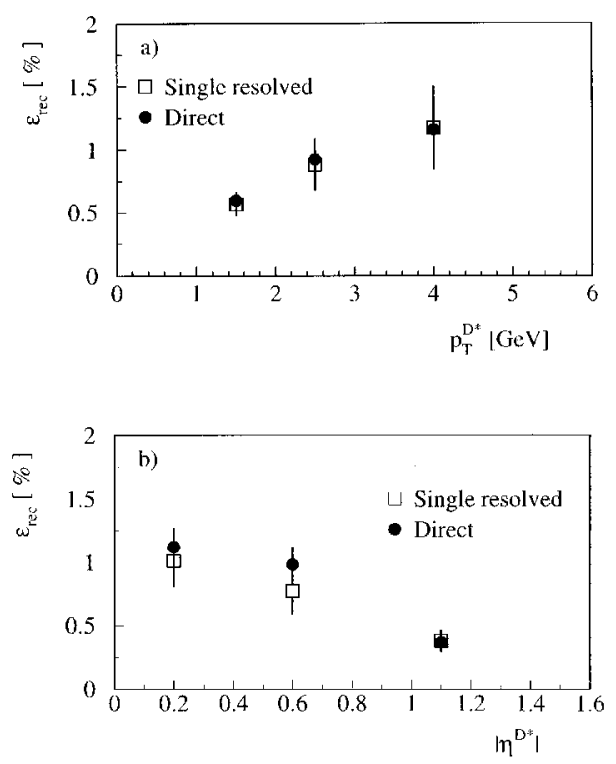

Fig. 3. Reconstruction efficiency of $\mathrm{D}^{*} \pm$ mesons (including the branching fractions), determined from PYTHIA generation of direct and single resolved-photon processes (a) as a function of $p_{T}^{\mathrm{D}^{*}}$ for $\left|\eta^{\mathrm{D}^{*}}\right|<1.4$ and (b) as a function of $\left|\eta^{\mathrm{D}^{*}}\right|$ for $1 \mathrm{GeV}<$ $p_{T}^{\mathrm{D}^{*}}<5 \mathrm{GeV}$.

program. A massive matrix element calculation with charmed quark mass value $m_{c}=1.35 \mathrm{GeV}$ and the SaS1d parametrization of the parton distributions of the photon [17] was used for the generation of events. The reconstruction efficiencies are calculated as a ratio of the combined number of reconstructed $\mathrm{D}^{* \pm}$ mesons in the two decay channels to the number of generated $\mathrm{D}^{*} \pm$ mesons and are presented in Fig. 3 as functions of $p_{T}^{\mathrm{D}^{*}}$ and $\left|\eta^{\mathrm{D}}\right|$. Evaluated in this way, the efficiencies take into account the corresponding branching fractions of the decay modes (1) and (2). The two sets of efficiencies are similar and agree within the errors. This implies that the relative

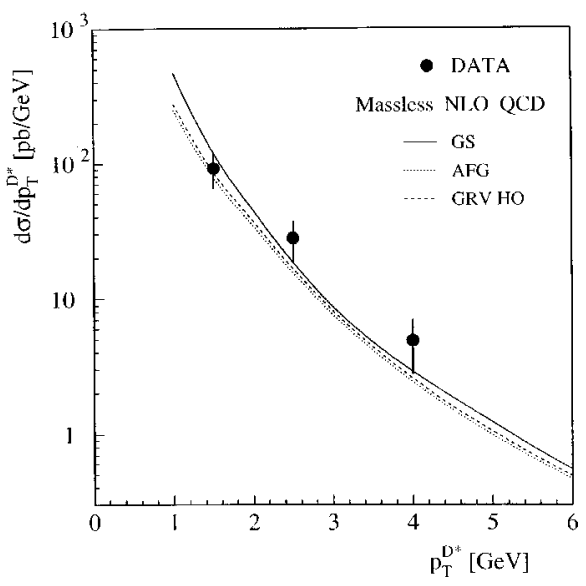

Fig. 4. The differential cross section of $D^{* \pm}$ production as a function of the transverse momentum of the $D^{*} \pm$ mesons for $\left|\eta^{D^{*}}\right|<1.4$. The points represent the data, the error bars show the statistical and systematic errors added in quadrature. The curves represent next-to-leading order QCD calculations [18] for different parameterizations of the parton densities of the photon (GS [19], AFG [20] and GRV-HO [21]).

proportion of direct and resolved-photon contributions to the charm production is not a major source of uncertainty in the determination of the $\mathrm{D}^{*} \pm$ differential cross sections in the phase space region defined by (3). Equal contributions of both types of charm production processes in the kinematic region (3) are assumed for the calculation of the reconstruction efficiencies, used for the cross-section evaluation.

The measured cross sections of inclusive $D^{*} \pm$ production, calculated as functions of $p_{T^{\prime}}^{\mathrm{D}^{*}}$ and $\left|\eta^{\mathrm{D}^{*}}\right|$ and integrated over the corresponding bin, are listed in Table 1. The differential cross sections, $d \sigma / d p_{T}^{\mathrm{D}}{ }^{*}$ and $d \sigma / d\left|\eta^{\mathrm{D}^{*}}\right|$, assigned to the bin centres, are plotted in Figs. 4 and 5. When evaluating the differential cross sections, a correction obtained with the

Table 1

Measured cross sections $\Delta \sigma_{\text {me as }}$ for inclusive $\mathrm{D}^{* \pm}$ production, integrated over the corresponding bin. The third and sixth columns of the table give the differential cross sections after bin-centre corrections. The first errors are statistical, the second systematic

\begin{tabular}{|c|c|c|c|c|c|}
\hline $\begin{array}{l}p_{T}^{\mathrm{D}^{*}} \\
\mathrm{GeV}\end{array}$ & $\begin{array}{l}\Delta \sigma_{\text {meas }} \\
\mathrm{pb}\end{array}$ & $\begin{array}{l}d \sigma / d p_{T}^{\mathrm{D}^{*}} \\
\mathrm{pb} / \mathrm{GeV}\end{array}$ & $\left|\eta^{D^{*}}\right|$ & $\begin{array}{l}\Delta \sigma_{\text {meas }} \\
\mathrm{pb}\end{array}$ & $\begin{array}{l}d \sigma / d\left|\eta^{\mathrm{D}^{*}}\right| \\
\mathrm{pb}\end{array}$ \\
\hline $1-2$ & $92.9 \pm 22.2 \pm 16.7$ & $92.9 \pm 22.2 \pm 16.7$ & $0.0-0.4$ & $34.1 \pm 8.4 \pm 5.3$ & $85 . \pm 21 . \pm 13$. \\
\hline $3-5$ & $11.3 \pm 3.9 \pm 3.0$ & $4.9 \pm 1.7 \pm 1.3$ & $0.8-1.4$ & $40.8 \pm 15.8 \pm 12.2$ & $68 . \pm 26 . \pm 20$ \\
\hline
\end{tabular}




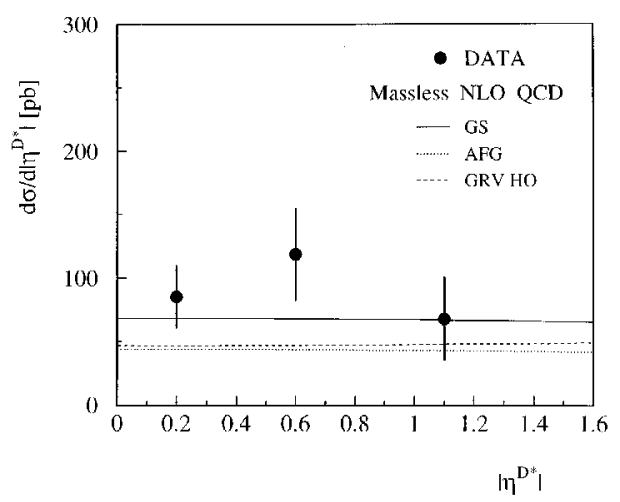

Fig. 5. The differential cross section of $D^{*} \pm$ production as a function of the pseudorapidity of the $\mathrm{D}^{* \pm}$ mesons for $1 \mathrm{GeV}<$ $p_{T}^{\mathrm{D}}$ " $<5 \mathrm{GeV}$. The points represent the data, the error bars show the statistical and systematic errors added in quadrature. The curves represent next-to-leading order QCD calculations as in Fig. 4.

combined Monte Carlo sample, used for the efficiency determination, is applied such as to assign the differential cross sections to the centres of the corresponding bins. The differential cross sections, obtained after applying the bin-centre correction, are also listed in Table 1.

The systematic uncertainties on the measured cross sections are estimated by varying the selection of tracks and photons and by varying the cuts throughout the $D^{* \pm}$ reconstruction. The contribution of the selection procedure to the systematic errors is in the range $8-17 \%$ affecting mostly the low $-p_{T}^{\mathrm{D}^{*}}$ region. The uncertainties in the $\mathrm{K}^{-} \pi^{+} \pi^{0}$ channel are higher than in the $\mathrm{K}^{-} \pi^{+}$channel. The systematic uncertainties related to the background estimation are determined by using different forms for the background function in the mass difference fit and by changing the mass range of the fit and are found to vary from $5 \%$ to $10 \%$. The $\mathrm{D}^{* \pm}$ reconstruction efficiencies are calculated also using a Monte Carlo sample generated by the JAMVG program which involves only direct processes in charm production, as well as for PYTHIA generations of direct and resolved processes with varied charmed quark mass value. The observed variations of the reconstruction efficiencies are taken into account as well as the Monte Carlo statistics, resulting in systematic changes of $5-14 \%$. The contributions of the various sources of systematic errors are added in quadrature.
The integrated cross section measured in the visible kinematic region is found to be ${ }^{10}$

$$
\begin{aligned}
& \sigma\left(\mathrm{e}^{+} \mathrm{e}^{-} \rightarrow \mathrm{e}^{+} \mathrm{e}^{-} \mathrm{D}^{*}{ }^{*} \mathrm{X} ; 1 \mathrm{GeV}<p_{T}^{\mathrm{D}^{*}}<5 \mathrm{GeV},\right. \\
& \left.\left|\eta^{\mathrm{D}^{*}}\right|<1.4\right) \\
& =132 \pm 22 \pm 26 \mathrm{pb},
\end{aligned}
$$

where the first error is statistical and the second systematic.

The integrated cross sections calculated separately for the $\mathrm{K}^{-} \pi^{+}$and $\mathrm{K}^{-} \pi^{+} \pi^{0}$ channels, $\sigma=124 \pm$ $24 \mathrm{pb}$ and $\sigma=142 \pm 46 \mathrm{pb}$ respectively (the errors are statistical only), agree well. This justifies combining the signals observed in the two decay modes.

In Fig. 4 and Fig. 5 the differential cross sections are compared to next-to-leading order perturbative QCD computations, based on a massless approach in calculating the parton-level cross sections [18]. In this scheme the charmed quark is considered to be one of the active flavours inside the photon. Three different sets of parton density parameterizations of the photon have been used in the calculations: GS [19], AFG [20] and GRV-HO [21]. The renormalization scale, $\mu_{R}$, and the factorization scale of the photon structure function, $\mu_{\mathrm{F}}$, have been taken as $\mu_{\mathrm{R}}=\mu_{\mathrm{F}} / 2=\sqrt{p_{T}^{2}+m_{c}^{2}}$ with charmed quark mass value $m_{c}=1.5 \mathrm{GeV}$ [18]. There is a reasonable agreement between the data and the calculations. With regard to the variations of the predictions in the region of low transverse momentum, we should notice the limited applicability of the massless approach for $p_{T} \approx m_{c}$ [22].

\section{Summary}

The inclusive production of $\mathrm{D}^{*} \pm$ mesons in two-photon interactions at LEP is measured by reconstructing $\mathrm{D}^{*+}$ cascade decays involving $\mathrm{D}^{0}$ decays into $\mathrm{K}^{-} \pi^{+}$and $\mathrm{K}^{-} \pi^{+} \pi^{0}$ final states, as well as the charge conjugate decay chains. The integrated and differential cross sections of inclusive $D^{*} \pm$

\footnotetext{
${ }^{10}$ The integrated cross section value is slightly different from the sum of partial cross sections, $\Delta \sigma_{\text {meas }}$, given in Table 1 , since the fits to the mass spectra and the efficiencies are evaluated independently in each bin.
} 
production are determined in the kinematic region $1 \mathrm{GeV}<p_{T}^{\mathrm{D}^{*}}<5 \mathrm{GeV},\left|\eta^{\mathrm{D}^{*}}\right|<1.4$ for which the acceptance is found to be insensitive to the relative mixture of direct and single resolved-photon processes. In this phase space domain the integrated cross section is measured to be $\sigma\left(\mathrm{e}^{+} \mathrm{e}^{-} \rightarrow\right.$ $\mathrm{e}^{+} \mathrm{e}^{-} \mathrm{D}^{*}{ }^{ \pm} \mathrm{X}$ ) $=132 \pm 22$ (stat.) \pm 26 (syst.) pb. A reasonable agreement is observed between the measured differential cross sections and the predictions based on next-to-leading order perturbative QCD calculations.

\section{Acknowledgements}

We express our gratitude to the CERN accelerator divisions for the excellent performance of the LEP machine. We also acknowledge and appreciate the effort of the engineers, technicians and support staff who have participated in the construction and maintenance of this experiment. We thank B.A. Kniehl for providing us with the results of QCD cross section calculations.

\section{References}

[1] M. Drees, M. Krämer, J. Zunft, P.M. Zerwas, Phys. Lett. B 306 (1993) 371.

[2] JADE Collaboration, W. Bartel, Phys. Lett. B 184 (1987) 288.
[3] TPC/Two-Gamma Collaboration, M. Alston-Garnjost et al., Phys. Lett. B 252 (1990) 499.

[4] TASSO Collaboration, W. Braunschweig, Z. Phys. C 47 (1990) 499.

[5] TOPAZ Collaboration, R. Enomoto et al., Phys. Lett. B 328 (1994) 535; Phys. Rev. D 50 (1994) 1879; Phys. Lett. B 341 (1994) 238; M. Iwasaki et al., Phys. Lett. B 341 (1994) 99

[6] VENUS Collaboration, S. Uehara, Z. Phys. C 63 (1994) 213.

[7] AMY Collaboration, T. Aso et al., Phys. Lett. B 363 (1995) 249; N. Takashimizu et al., Phys. Lett. B 381 (1996) 372.

[8] ALEPH Collaboration, D. Buskulic, Phys. Lett. B 3555 (1995) 595.

[9] L3 Collaboration, M. Acciarri, Phys. Lett. B 453 (1999) 83.

[10] S. Nussinov, Phys. Rev. Lett. 35 (1975) 1672; G.J. Feldman et al., Phys. Rev. Lett. 38 (1977) 1313.

[11] Particle Data Group, C. Caso, Eur. Phys. J. C 3 (1998) 34.

[12] L3 Collaboration, B. Adeva et al., Nucl. Instr. Meth. A 289 (1990) 35; M. Acciarri et al., Nucl. Instr. Meth. A 351 (1994) 300; M. Chemarin et al., Nucl. Instr. Meth. A 349 (1994) 345; I.C. Brock et al., Nucl. Instr. Meth. A 381 (1996) 236; A. Adam et al., Nucl. Instr. Meth. A 383 (1996) 342.

[13] T. Sjöstrand, CERN-TH/7112/93, 1993, revised August 1995; T. Sjöstrand, Comp. Phys. Comm. 82 (1994) 74.

[14] J.A.M. Vermaseren, Nuc. Phys. B 229 (1983) 347.

[15] S. Jadach, B.F.L. Ward, Z. Was, Comp. Phys. Comm. 79 (1994) 503

[16] M. Skrzypek et al., Comp. Phys. Comm. 94 (1996) 216; Phys. Lett. B 372 (1996) 289.

[17] G.A. Schuler, T. Sjöstrand, Z. Phys. C 68 (1995) 607; Phys. Lett. B 376 (1996) 193.

[18] J. Binnewies, B.A. Kniehl, G. Kramer, Phys. Rev. D 53 (1996) 6110; J. Binnewies, B.A. Kniehl, G. Kramer, Phys. Rev. D 58 (1998) 014014; B.A. Kniehl, private communication.

[19] L.E. Gordon, J.K. Storrow, Nuc. Phys. B 489 (1997) 405.

[20] P. Aurenche, J.-P. Guillet, M. Fontannaz, Z. Phys. C 64 (1994) 621.

[21] M. Glück, E. Reya, A. Vogt, Phys. Rev. D 46 (1992) 1973

[22] S. Frixione, Phys. Lett. B 319 (1993) 339. 